\title{
Growth hormone receptor inhibition decreases the growth and metastasis of pancreatic ductal adenocarcinoma
}

\author{
Ramadevi Subramani, Rebecca Lopez-Valdez, Alyssa Salcido, Thiyagarajan Boopalan, Arunkumar Arumugam, \\ Sushmita Nandy and Rajkumar Lakshmanaswamy
}

Pancreatic cancer is the only major cancer with very low survival rates (1\%). It is the fourth leading cause of cancer-related death. Hyperactivated growth hormone receptor (GHR) levels have been shown to increase the risk of cancer in general and this pathway is a master regulator of key cellular functions like proliferation, apoptosis, differentiation, metastasis, etc. However, to date there is no available data on how GHR promotes pancreatic cancer pathogenesis. Here, we used an RNA interference approach targeted to GHR to determine whether targeting GHR is an effective method for controlling pancreatic cancer growth and metastasis. For this, we used an in vitro model system consisting of HPAC and PANC-1 pancreatic cancer cells lines. GHR is upregulated in both of these cell lines and silencing GHR significantly reduced cell proliferation and viability. Inhibition of GHR also reduced the metastatic potential of pancreatic cancer cells, which was aided through decreased colony-forming ability and reduced invasiveness. Flow cytometric and western blot analyses revealed the induction of apoptosis in GHR silenced cells. GHR silencing affected phosphatidylinositol 3 kinase/AKT, mitogen extracellular signal-regulated kinase/extracellular signalregulated kinase, Janus kinase/signal transducers and activators of transcription and mammalian target of rapamycin signaling, as well as, epithelial to mesenchymal transition. Interestingly, silencing GHR also suppressed the expression of insulin receptor- $\beta$ and cyclo-oxygenease-2. Altogether, GHR silencing controls the growth and metastasis of pancreatic cancer and reveals its importance in pancreatic cancer pathogenesis.

Experimental \& Molecular Medicine (2014) 46, e117; doi:10.1038/emm.2014.61; published online 10 October 2014

\section{INTRODUCTION}

Cancer of the pancreas occurs most often in cells of the exocrine compartment, which accounts for $95 \%$ of all pancreatic cancer cases. In particular, pancreatic ductal adenocarcinoma (PDAC) is the most common type $(90 \%)$ of pancreatic cancer diagnosed in the exocrine component of the pancreas. Pancreatic cancer incidence and mortality rate are nearly equivalent and the 5-year survival rate is estimated at only $1 \% .{ }^{1}$ This makes the pancreatic cancer one of the most lethal malignant carcinomas affecting humans. ${ }^{2}$ This dismal prognosis is due to absence of early warning signs and symptoms. ${ }^{3}$ This is also due to PDAC resistance/ unresponsiveness to existing conventional treatments like chemotherapy and radiation therapy. ${ }^{2}$ These characteristics coupled with the highly invasive nature of PDAC translate to a median survival rate of $<6$ months in most pancreatic cancer patients. ${ }^{2}$
Most PDAC cases (99\%) are reported to arise from KRAS gene mutation. ${ }^{4}$ However, this known genetic information has not yet led to the development of effective targeted therapeutic strategies. This indicates that there is still much lacking in understanding the molecular mechanisms involved in PDAC pathogenesis. Growth hormone receptor (GHR), a member of the cytokine receptor superfamily, has been shown to regulate multiple cellular pathways including KRAS and insulin-like growth factor-1 receptor (IGF-1R) signaling. ${ }^{5}$ In fact, an increased level of GHR in pancreatic cancer cells has been demonstrated in earlier studies. ${ }^{6}$ Therefore, GHR could serve as a valid target to study in pancreatic cancer tumorigenesis because it has a direct role in KRAS signaling and is aberrantly expressed in a majority of cancer types, including pancreatic cancer.

Growth hormone (GH) activates mitogenic signaling through Janus kinase (JAK)/signal transducers and activators

Center of Excellence in Cancer Research, Department of Biomedical Sciences MSB1, Texas Tech University Health Sciences Center, Paul L Foster School of Medicine, El Paso, TX, USA

Correspondence: Dr R Lakshmanaswamy, Center of Excellence in Cancer Research, Department of Biomedical Sciences MSB1, Texas Tech University Health Sciences Center, Paul L Foster School of Medicine, 5001 El Paso Drive, El Paso, TX 79905, USA.

E-mail: rajkumar.lakshmanaswamy@ttuhsc.edu

Received 11 April 2014; revised 12 July 2014; accepted 31 July 2014 
of transcription (STAT), mitogen-activated protein kinase (MAPK)/extracellular signal-regulated kinase (ERK) and mammalian target of rapamycin (mTOR) pathways. ${ }^{7}$ GHR is also known to mediate its effects via IGF-1R. ${ }^{8}$ IGF-1R overexpression has often been noted in pancreatic cancer and has been tied to resistance to apoptosis, increased growth and survival, etc., of pancreatic cancer. ${ }^{9}$ Thus, there is clear evidence that the GH/IGF1 signaling axis is involved in the development and progression of pancreatic cancer, as well as, many other cancer types. ${ }^{10}$ However, the molecular mechanisms and signaling pathways responsible are only beginning to be unraveled. In fact, the role of GHR in pancreatic cancer has not yet been well studied. To our knowledge, this is the first study assessing the role of GHR in PDAC, revealing for the first time the importance of GHR in pancreatic tumorigenesis.

\section{MATERIALS AND METHODS}

\section{Ethics statement}

All the experiments performed were approved and performed following the guidelines of the Institutional Biosafety Committee of Texas Tech University Health Sciences Center.

\section{Cell lines, reagents and antibodies}

PDAC cell lines such as PANC-1, HPAC and MIAPaCa-2 were purchased from the American Type Culture Collection (ATCC, Manassas, VA, USA); PANC-1 and HPAC cells were maintained in RPMI-1640 media supplemented with $10 \%$ fetal bovine serum, 100 units $\mathrm{ml}^{-1}$ of penicillin and $100 \mu \mathrm{g} \mathrm{ml}^{-1}$ of streptomycin. MIAPaCa-2 cells were maintained in Dulbecco's modified Eagle's medium supplemented with $10 \%$ FBS and $2.5 \%$ horse serum. Normal human pancreas cell line hTERT-HPNE (CRL-4023) was obtained from ATCC and maintained in 75\% Dulbecco's modified Eagle's medium and 25\% M3 base medium supplemented along with 5\% FBS, 10 ng ml${ }^{-1}$ human recombinant epidermal growth factor, $5.5 \mathrm{~mm} \mathrm{D}$ glucose and $750 \mathrm{ng} \mathrm{ml}^{-1}$ puromycin. Cells were maintained at $37^{\circ} \mathrm{C}$ in a humidified atmosphere with $5 \%$ carbon dioxide.

TransIT- siQUEST transfection reagent was obtained from Mirus Bio (Madison, WI, USA). Transwell plate inserts $(6.5 \mathrm{~mm}, 8.0 \mu \mathrm{m}$ pore polycarbonate membrane) were purchased from Corning Incorporated (Corning, NY, USA). BD Pharmingen Annexin V-FITC Apoptosis Detection Kit I (San Diego, CA, USA) and BD Matrigel was purchased from BD Biosciences (Bedford, MA, USA). GHR small interfering RNA (siRNA) targeting and GHR gene was obtained from Origene (Rockville, MD, USA). Bovine serum albumin was procured from Sigma-Aldrich Corporation (St Louis, MO, USA). Mammalian protein extraction reagent was acquired from Thermo Scientific (Rockford, IL, USA). Promega's (Madison, WI, USA) MTS reagent [3-(4, 5- dimethylthiazol-2-yl)-5-(3-carboxymethoxyphenyl)-2-(4-sulfophenyl)-2H-tetrazolium] was used for cell viability assays.

Antibodies used in this study are as follows: STAT3 (H-190), pERK (sc-101760), ERK (sc-94) (sc-7179), AKT (5298), pAKT (sc-101629) and Bcl-2 (sc-783) were acquired from Santa Cruz Biotechnology (Santa Cruz, CA, USA). IR- $\beta$ (3024), pIRS-1(2388), IRS-1(2382), pSTAT3 (ser 727) (4113), pPI3K p85(4228), PI3K p85(4292), pPTEN (9549), pmTOR(2974), mTOR(4517), pp70s6kinase (9206), p70s6kinase (9202), Bax (2772), caspase 3 (9661), PARP (9542), Notch 2 (4530P), Snail (3879), E-cadherin (3195), N-cadherin (4061), Zeb (3396), Vimentin (5741), Slug (9585), IGF-1R(3027), Ras(3965) and COX-2 (4842) were acquired from Cell Signaling Technology (Boston,
MA, USA). GHR (ab 78426) and caspase 8 (ab 25901) antibodies was purchased from Abcam (Cambridge, MA, USA). $\beta$-Actin was procured from Sigma-Aldrich Corporation. Appropriate secondary antibodies were obtained from Santa Cruz Biotechnology.

\section{Expression of GHR in human pancreas adenocarcinoma}

Immunohistochemistry was done to determine the expression levels of GHR in human pancreas adenocarcinoma tissue microarray (TMA); obtained from US Biomax, Inc., Rockville, MD, USA. The TMA contains formalin-fixed paraffin-embedded samples of normal pancreatic and pancreatic adenocarcinoma tissues with varying tumor stages (stages 1-4). Institutional Review Board approval was not required for using TMAs.

Immunohistochemistry for GHR antigen was performed using the pancreas adenocarcinoma TMA. Tissue adhesion was enhanced by incubating the charged glass slide at $58^{\circ} \mathrm{C}$ for $2 \mathrm{~h}$. Deparaffinization and rehydration was achieved by xylene and serial ethanol baths (100, 95, 70, 50 and 30\%), respectively. Heat-induced epitope retrieval with trilogy (Cell Marque, Rocklin, CA, USA) was then done to unmask the antigenic sites. Perox-free blocking reagent (Cell Marque) was added to inhibit nonspecific antibody binding. TMAs were incubated with GHR antibody (1:50 dilution) overnight at $4{ }^{\circ} \mathrm{C}$. Ultra Marque polyscan HRP Label (Cell Marque) was then added for $1 \mathrm{~h}$. After phosphate-buffered saline wash, TMAs were stained with the chromogen solution (Cell Marque) for $20 \mathrm{~min}$. The TMAs were then counterstained with hematoxylin for $40 \mathrm{~s}$. The TMAs were dehydrated with serial ethanol baths (30,50, 70, 95 and 100\%) followed by a xylene bath. Finally, TMAs were mounted with the mounting media (Surgipath Medical Industries, Richmond, IL, USA) and digital images were obtained using a Nikon Microscope- ECLIPSE 50i (Brighton, MI, USA).

\section{Transfections of GHR siRNA in PANC-1 and HPAC cells} siRNA targeting GHR was transiently transfected into PANC-1 and HPAC cells using MIrus bio TransIT siQUEST transfection reagent. A non-targeting siRNA sequence (Scrambled) was used as a control. In brief, cells were seeded at a density of $2.5 \times 10^{5}$ cells per well in six-well plates. Cells were transfected with different siRNA subtypes (A, B and C) at $10 \mathrm{~nm}$ concentration for $48 \mathrm{~h}$ using Mirus siQUEST transfection reagent. According to the manufacturer's protocol, siRNA and transfection reagent ratio was maintained as 1:0.5 for efficient silencing without toxicity. The $10 \mathrm{~nm}$ concentration of siRNAs was found to be sufficient for maximum silencing of GHR in both the cell lines. After $48 \mathrm{~h}$, transfected cells were used for protein isolation or invasion, migration, clonogenicity or apoptosis studies. To assess the biological effect of GHR in pancreatic carcinogenesis, GHR silenced PANC-1 and HPAC were used for all the experiments.

\section{Cell viability assay}

PANC-1 and HPAC cells were transfected with GHR siRNA at a final concentration of $10 \mathrm{~nm}$ for $48 \mathrm{~h}$ along with scrambled controls. For this assay, PANC-1 and HPAC cells were seeded in 96-well plates at a density of $0.3 \times 10^{4}$ cells per well and $0.5 \times 10^{4}$ cells per well, respectively. After $48 \mathrm{~h}$, the MTS assay was performed as per the manufacturer's instructions. Absorbance at $490 \mathrm{~nm}$ was quantified on the Victor X4 2030 plate reader (Perkin Elmer, Waltham, MA, USA).

\section{Colony formation assay}

PANC-1 and HPAC cells were transfected with GHR siRNA as described above. Transfected cells were then seeded at a density of 
$2 \times 10^{4}$ cells in $60-\mathrm{mm}$ dishes containing a top layer of $0.7 \%$ agar and a bottom layer of $1 \%$ agar. Cells were incubated at $37^{\circ} \mathrm{C}$ until visible colonies formed (approximately 3-4 weeks). Colonies were then stained with $0.2 \%$ crystal violet and visible colonies were counted manually in five separate fields per dish.

\section{Scratch assay}

Cell migration in GHR silenced cells was measured via scratch assay technique. Transfected cells were seeded in six-well plates at a density of $3.5 \times 10^{5}$ cells per well and allowed to reach monolayer confluence. At this time, a scratch was gently made with a sterile pipette tip in each monolayer culture. All detached cells were removed with phosphatebuffered saline wash; cell monolayers were then replenished with fresh media and monitored continuously for $96 \mathrm{~h}$ using the Biostation CT (Nikon Instruments Inc., Melville, NY, USA). Photographs were automatically captured every $2 \mathrm{~h}$ over a period of $96 \mathrm{~h}$. The resulting migration images were recorded and analyzed using NIS-Element AR software (Melville, NY, USA).

\section{In vitro cell migration and invasion assay}

GHR silenced PANC-1 and HPAC cells were trypsinized and resuspended in FBS-free RPMI-1640 media. Migration assay was performed using a total of $5 \times 10^{3}$ cells in the top chamber of the transwell with a noncoated polycarbonate membrane. Similarly, the invasion assay was executed by seeding $2 \times 10^{4}$ cells in the top chamber of the transwell polycarbonate membrane coated with matrigel at a concentration of $1 \mathrm{mg} \mathrm{ml}^{-1}$. RPMI-1640 media with $10 \%$ FBS was used as a chemoattractant in the lower chamber. Migrating and invading cells on the lower surface of the membrane were fixed with $5 \%$ formalin and stained with $0.2 \%$ crystal violet after 48 - $\mathrm{h}$ incubation. Images of migratory and invading cells were captured using the Nikon Eclipse TS 100 microscope at $\times 20$ magnification. Cells were then counted in a blinded manner at five or six randomly selected fields.

\section{In vitro assays for cell death using flow cytometry (apoptosis assay)}

Annexin V-FITC Apoptosis Detection Kit I was used to assess the apoptotic effect of GHR silencing in pancreatic cancer cells. Fortyeight hours post-transfection, cells were harvested and stained with Annexin $\mathrm{V}$-fluorescein isothiocyanate and propidium iodide according to the manufacturer's instructions. The percentage of apoptosis or cell death was assessed using a flow cytometer (FACS Accuri C6, San Jose, CA, USA).

\section{Immunoblot analysis}

Equal amounts of protein were separated by sodium dodecyl sulfatepolyacrylamide gel electrophoresis and transferred onto polyvinylidene difluoride membranes. Membranes were blocked with 5\% bovine serum albumin in $1 \mathrm{X}$ Tris-buffered saline with Tween 20 and were probed overnight with a panel of primary antibodies against pAKT, AKT, Bcl-2, pERK, ERK, STAT3, IGF-1R, Notch 2, Snail, E-cadherin, N-cadherin, Zeb, Vimentin, Slug, Bax, caspase 3, PARP, pPI3K p85, PI3K p85, IR- $\beta$, pIRS- 1 , IRS- 1 , pSTAT3, COX-2, pPTEN, pmTOR, mTOR, pp70s6kinase, p70s6kinase, caspase 8 and $\beta$-actin. Proteins of interest were detected with secondary horseradish peroxidase-coupled antibodies and visualized using enhanced chemiluminescence.

\section{Statistical analysis}

Statistical analysis was conducted using Prism version 5.03 software (La Jolla, CA, USA). Unpaired Student's $t$-test was applied to determine significant differences between groups. The $P$-value $<0.05$ was regarded as significant, whereas $P$-values $<0.01$ and 0.001 were regarded as highly significant.

\section{RESULTS}

Aberrant expression of GHR in PDAC cell lines

GHR has a vital role in carcinogenesis, which has been clearly demonstrated in metastatic melanoma where GHR expression is 50 -fold higher than in normal cells. However, the precise role of GHR in the carcinogenesis of many other cancers, including pancreatic cancer, remains to be defined. ${ }^{11}$ To the best of our knowledge, ours is the first study to examine GHR expression and function in PDAC cell lines. Here, we detected higher expression levels of GHR in all three aggressive pancreatic cell lines, PANC-1, HPAC, and MIAPaCa-2 when compared with normal pancreatic cell line hTERT HPNE (Figure 1a). PANC-1 had the highest expression of GHR, whereas HPAC had the least expression among the three cell line tested. So we chose to use PANC1 and HPAC cells for all our experiments based on their GHR expression (Figure 1a). This result suggests a prominent role for GHR in pancreatic carcinogenesis.

Immunohistochemistry analysis was performed to further confirm the expression levels of GHR in normal pancreatic tissue and pancreatic adenocarcinomas. GHR expression increased with increasing grade of PDAC (Figure 1b).

\section{GHR silencing by predesigned siRNA induces anti- proliferative effect in PDAC cell lines}

GHR was silenced in PANC-1 and HPAC cells using predesigned siRNAs at a concentration of $10 \mathrm{~nm}$ (Figure 1c). As GHR has direct as well as indirect effects on cell proliferation through modulation of various intracellular signaling pathways, ${ }^{12}$ we next examined the effect of GHR silencing on cell viability. PANC-1 and HPAC cells were transfected with GHR siRNA (si-GHR) or scrambled control (Scr) for $48 \mathrm{~h}$ and cell viability was assessed using MTS assay. Cell viability was notably attenuated in GHR silenced cells with only $39.6 \%$ and $21.5 \%$ viable cells in PANC1 and HPAC cells, respectively (Figure 1d). GHR silencing significantly increases cell death, which clearly indicates the proliferative effect of GHR in pancreatic cancer cells.

\section{Silencing GHR inhibits soft agar colony formation}

To assess anchorage-independent growth potential of GHR silenced PANC-1 and HPAC cells, they were subjected to the soft agar clonogenic assay-a well-known technique for assessing anchorage-independent growth and thus metastatic properties of cells. GHR knockdown drastically reduced the number and size of colonies for both PANC-1 and HPAC cells (Figure 1e). Results from three independent experiments were quantified revealing $>90 \%$ colony-forming inhibition in GHR silenced pancreatic cancer cells (Figure 1f). Our result 
a

Elevated GHR expression levels

Normal PANC-1 HPAC MIAPaCa-2

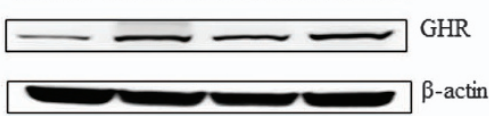

C

Silencing GHR using siRNA Technology

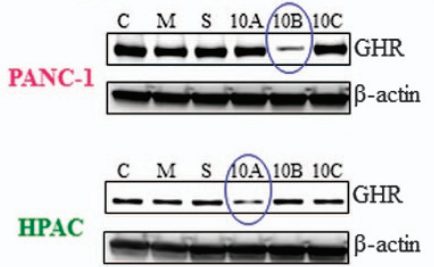

b

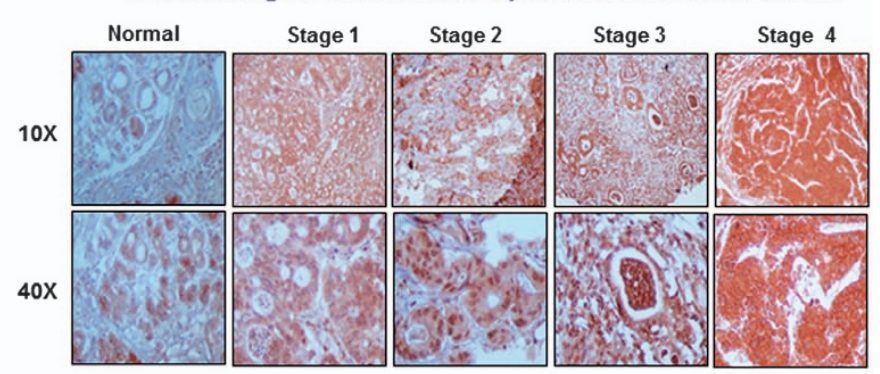

d

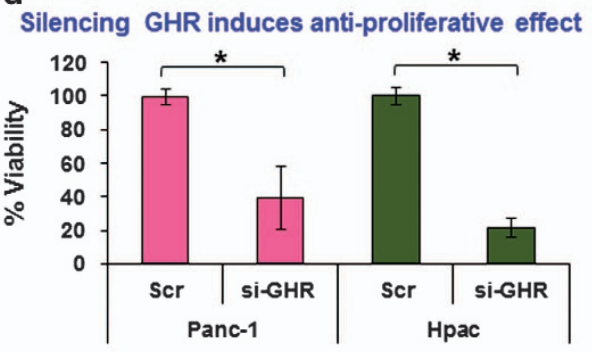

f

Graphical representation of colony forming assay

Inhibition of GHR expression blocks colony forming capabilities
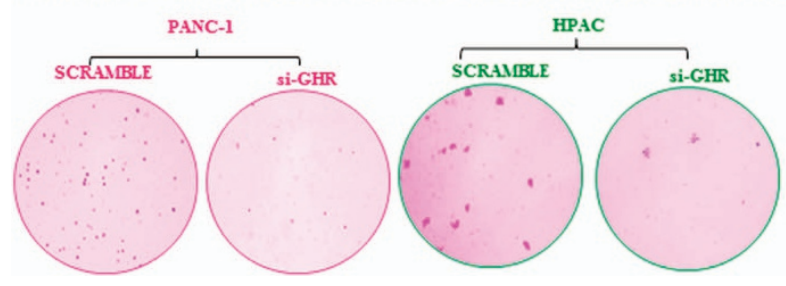

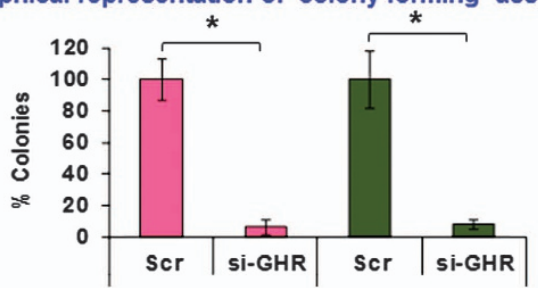

Figure 1 Silencing growth hormone receptor (GHR) inhibited viability and anchorage-independent growth of pancreatic ductal adenocarcinoma (PDAC) cell lines. (a) GHR expression levels from PANC-1 HPAC and MIAPaCa-2 were compared with normal pancreatic cells using western blot. (b) Representative immunohistochemical analysis of GHR by tumor stage in pancreatic adenocarcinoma tissues and normal pancreas tissue. (c) Three predesigned GHR small interfering RNAs (siRNAs) (a-c) were transfected in PANC-1 and HPAC cells at $10 \mathrm{~nm}$ along with scrambled control siRNA (labeled as 'S'), untreated control PANC-1 and HPAC cells (labeled as 'C') and mock control transfection reagent alone (labeled as ' $M$ '). GHR siRNA silencing efficacy was examined using western blot in PANC-1 and HPAC cells. (d) Effect of GHR knockdown on cell viability of PANC-1 and HPAC. PANC-1 and HPAC cells were transfected with 10 nm of GHR siRNA and scrambled siRNA. MTS assay kit was used to determine the cell viability of post transfected cells after $48 \mathrm{~h}$. Error bars denote the s.d. between triplicates. (e) Loss of anchorage-independent growth in GHR silenced PDAC cells, PANC-1 and HPAC. Colony-forming abilities of GHR transfected PANC-1 and HPAC were determined using soft agar colony formation assay along with scrambled control. Transfected PANC-1 and HPAC cells were allowed to grow in $0.7 \%$ agarose in growth media supplemented $10 \%$ fetal bovine serum (FBS) for 16 and 28 days, respectively. Representative images of colony formation were shown from two independent experiments done in triplicate. (f) Percentage of colonies in transfected PANC-1 and HPAC cells were compared with scrambled control (SCR) serving as the baseline. Data presented as the mean \pm s.d. $(n=3)$. ${ }^{*} P<0.05$.

shows that GHR has a key role in anchorage-independent growth and metastatic properties of pancreatic cancer cells (Figure 1f).

\section{Inhibition of cell migration/motility by GHR suppression}

(i) Scratch assay/wound-healing assay. Colony-forming ability and migration/invasion capabilities are characteristics generally associated with cancer cell metastasis. ${ }^{13}$ The classic scratch assay was performed to study the role of GHR in regulating the migratory ability of pancreatic cancer cells. After GHR knockdown, a scratch was created in monolayer cultures and cell migration into the scratch was monitored over a period of $96 \mathrm{~h}$. Compared with scrambled controls, GHR suppressed PANC-1 and HPAC cells demonstrated reduced migratory abilities. Control PANC-1 cells reformed a complete monolayer within $96 \mathrm{~h}$ and control HPAC cells reformed a complete monolayer within $48 \mathrm{~h}$. Meanwhile, GHR suppressed cells did not reform a complete monolayer even after $96 \mathrm{~h}$ (Figures 2a-c).

(ii) Transwell migration assay. Suppressed migratory capabilities after GHR knockdown were further confirmed using transwell assays. Once again, compared with controls, both cell lines showed a marked reduction of migratory ability upon 


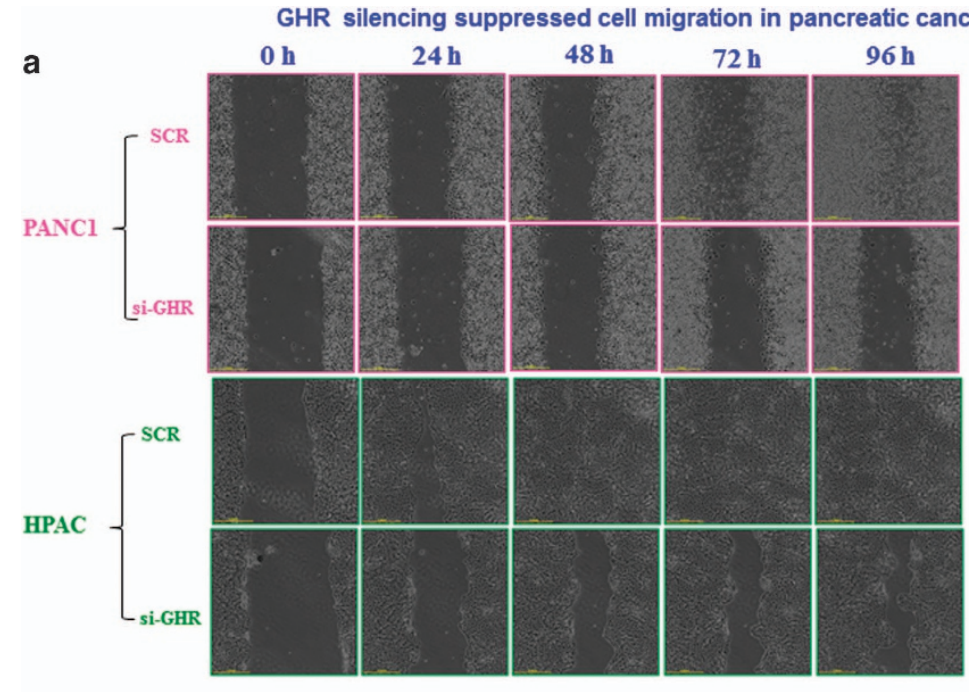

Silencing GHR expression inhibits migration (Transwell assay)
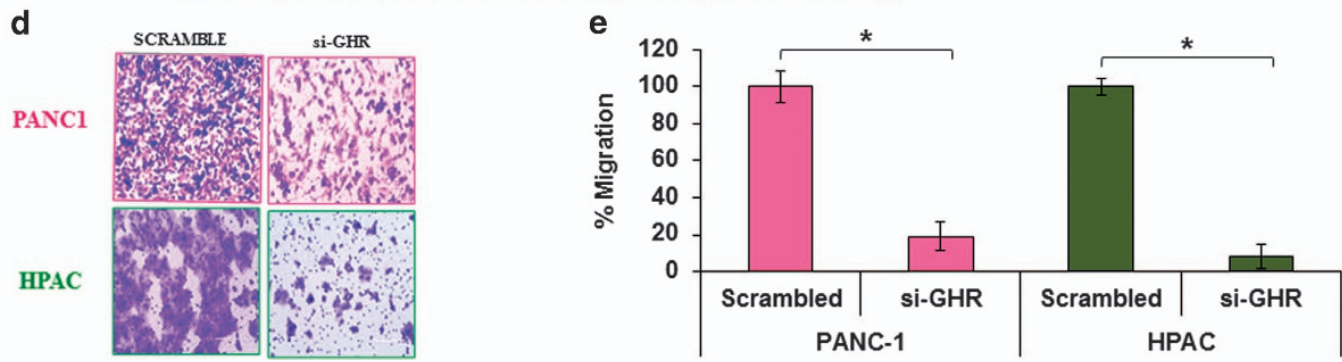

Figure 2 Silencing of growth hormone receptor (GHR) suppresses cell motility and migration in pancreatic ductal adenocarcinoma (PDAC) cell lines. (a) PANC-1 and HPAC cells were transfected with scrambled or GHR small interfering RNA (siRNA) and were performed wound healing assays with a 96-h recovery period. Forty-eight hours after post-transfection, monolayer cultured cells were scratched and cell motility was monitored with automated Nikon Biostation CT at 2-h intervals up to $96 \mathrm{~h}$. (b, c) The cell motility was analyzed by the rate of cells moving toward the scratched area. The percentage motility was calculated with the aid of NIS-Element AR software. Scr, Scrambled control siRNA and si-GHR, GHR siRNA treatment. (d) Knockdown of GHR inhibits migration of PANC-1 and HPAC cells. Transwell migration assay with matrigel uncoated Boyden chambers determined the cell migratory ability of GHR post transfected PANC-1 and HPAC cells. Post transfected PANC-1 and HPAC cells were incubated for $48 \mathrm{~h}$ to migrate through pores to the bottom surface of transwell. Migrated cells at the bottom of transwell were fixed and stained with $0.2 \%$ crystal violet in $5 \%$ formalin. (e) Graphical representation of percentage migration for transwell assays. Data presented as the mean \pm s.d. $(n=3)$. ${ }^{*} P<0.05$.

GHR silencing (Figures $2 \mathrm{~d}$ and e). The above results indicate the regulatory role of $\mathrm{GHR}$ in migration of pancreatic cancer cells.

\section{Knockdown of GHR inhibits cell invasion}

Invasion and metastasis are integral for tumor cell escape from the primary site of origin and dissemination into distant organs via blood and lymphatic systems. ${ }^{14}$ Matrigel-coated Boyden chamber inserts were designed to mimic the internal basement membrane of the tumor microenvironment to investigate the invasive potential of pancreatic cancer cells with and without GHR silencing. This assay clearly demonstrates reduced invasion by GHR silenced cells (Figure 3a). Compared with scrambled controls, GHR silenced cells exhibited a reduction of invasive capacity of $>82 \%$ and $91 \%$ in PANC- 1 and HPAC cells, respectively (Figure $3 \mathrm{~b}$ ). Collectively, these results indicate that silencing GHR decreases both migratory ability and invasive properties of PDAC cells.

\section{Silencing GHR inhibits EMT}

Epithelial to mesenchymal transition (EMT) is a well-known biological process that is often activated during invasion and metastasis of cancer. ${ }^{15}$ Here, we show that silencing GHR resulted in a reversal of EMT progression by suppressing the expression of proteins that drive the EMT process. Using western blot, we observed the inhibition of mesenchymal markers N-cadherin, vimentin, Notch-2, Snail, Zeb and slug in GHR knockdown cells (Figure 3c). Interestingly, expression levels of the epithelial marker E-cadherin were concurrently increased in GHR silenced cells (Figure 3c). E-cadherin is a key cell-cell adhesion molecule; loss of its expression is shown to be involved in metastasis. ${ }^{16}$ Densitometric analysis further confirmed that GHR silencing significantly represses the expression of proteins involved in EMT (Figure 3d).

\section{GHR silencing induces apoptosis}

Using Annexin V/propidium iodide staining, we demonstrate that GHR silencing results in a significant increase in apoptosis 

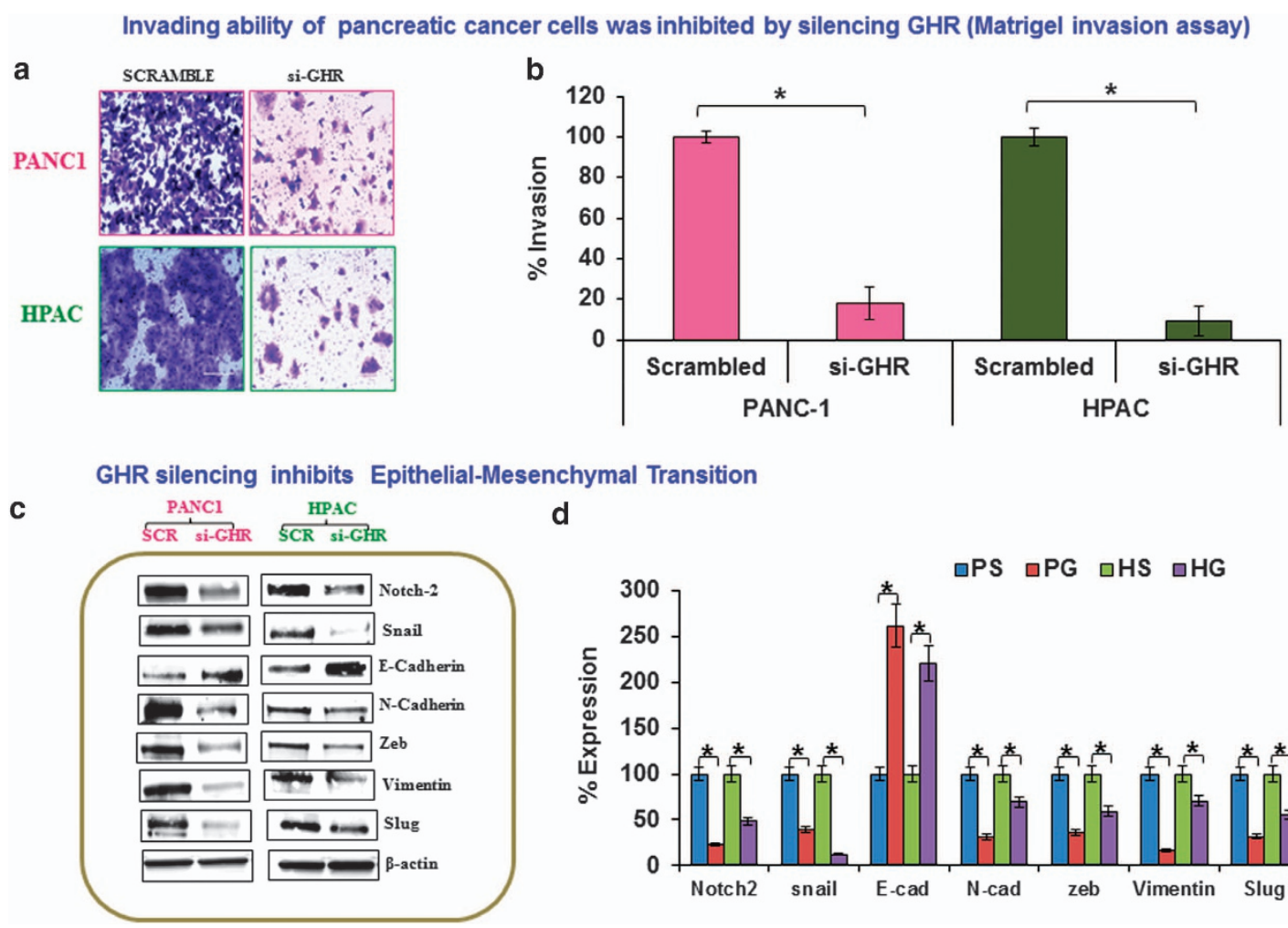

d

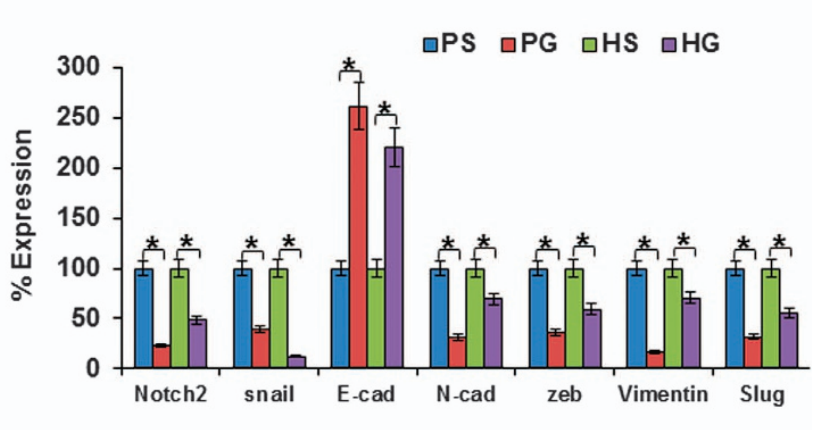

Figure 3 Silencing growth hormone receptor (GHR) inhibited invasion and metastasis through epithelial to mesenchymal transition (EMT). (a) Matrigel invasion assay was performed in GHR transfected PANC-1 and HPAC. Cells that had invaded through matrigel gel-coated Boyden chamber were fixed and stained with crystal violet. (b) Cells that invaded were counted and expressed as percentage of invasion. (c) EMT markers were inhibited in GHR silenced PANC-1 and HPAC cells. Protein expression levels of Notch-2, Snail, E-cadherin, $\mathrm{N}$-Cadherin, Zeb, Vimentin and Slug along with internal control $\beta$-actin were analyzed by western blotting in scrambled control and GHR silenced PANC-1 and HPAC cells. (d) Percentage expression of proteins was calculated using densitometric values obtained from density ratio of EMT markers/ $\beta$-actin. PS-PANC-1 Scrambled, PG-PANC-1 GHR silenced, HS-HPAC Scrambled, HG-HPAC GHR silenced. Data presented as the mean \pm s.d. $(n=3) .{ }^{*} P<0.05$.

in pancreatic cancer cells $(60.1 \%$ and $47.3 \%$ in PANC-1 and HPAC, respectively; Figures $4 \mathrm{a}$ and $\mathrm{b})$. To identify the molecular mechanism involved in the induction of apoptosis, we assessed the expression of key apoptotic molecules. In HPAC cells, GHR silencing resulted in increased expression of pro-apoptotic molecule Bax, caspase 8, caspase 3 and PARP. In PANC-1 cells, GHR silencing also results in increased expression of Bax, caspase 3 and PARP except caspase 8 . The antiapoptotic protein $\mathrm{Bcl}-2$ was downregulated in both PANC-1 and HPAC cells upon GHR silencing (Figures $4 \mathrm{c}$ and d). These data reveal that GHR silencing induces apoptosis via both death receptor and mitochondrial-mediated pathways of apoptosis in HPAC cells. Meanwhile, apoptosis is only mediated via the mitochondrial pathway in PANC-1 cells.

\section{Effect of GHR on key signaling pathways}

Finally, using western blot, we conducted a more comprehensive analysis of the molecular pathways affected by GHR silencing in pancreatic cancer cells. Our findings reveal that GHR does in fact coordinate the regulation of multiple cellular pathways involved in survival, proliferation, metastasis, EMT, apoptosis and cell cycle (Figures 5 and 6).
GHR silencing significantly downregulated the active forms of both AKT and phosphatidylinositol 3 kinase (PI3K), while total protein levels remained unchanged (Figures $5 \mathrm{a}-\mathrm{d}$ ). The $\mathrm{PI} 3 \mathrm{~K} / \mathrm{AKT}$ signaling pathway is frequently overexpressed in many cancers where it results in enhanced cell proliferation and reduced apoptosis. ${ }^{17}$

AKT/PI3K signaling activates mTOR and its downstream effector molecule pp70s6kinase. Therefore, we assessed the expression of these signaling intermediates in GHR silenced cells and found that mTOR phosphorylation is significantly inhibited in PANC-1 and HPAC cells, whereas total mTOR levels are increased upon GHR knockdown (Figures 5e and f). GHR silencing also decreased the phosphorylation of p70s6kinase, with no change observed in the expression of total p70s6kinase for PANC-1 cells and increased expression of total p70S6kinase in HPAC cells (Figures 5e and f).

$\mathrm{PI} 3 \mathrm{~K}$ is negatively regulated by a potent inhibitor phosphatase and tensin homolog (PTEN), which is mutated at high frequency in several cancers, including pancreatic cancer. ${ }^{18}$ Here, we found that PTEN was highly activated upon GHR silencing in both PANC-1 and HPAC cells (Figures 5e and f). Activation of PTEN in GHR silenced cells efficiently enhances 
GHR inhibition induces Apoptosis in PANC-1 \& HPAC cells

a

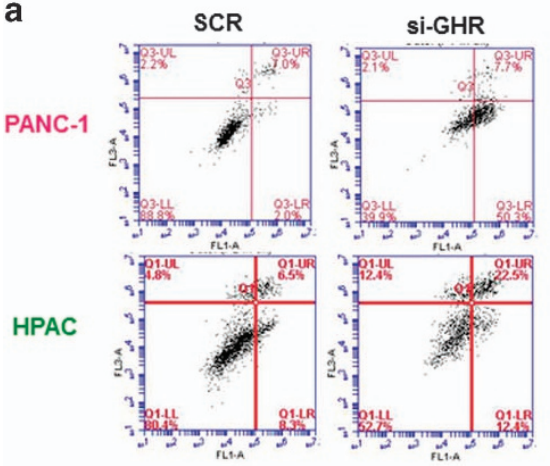

b

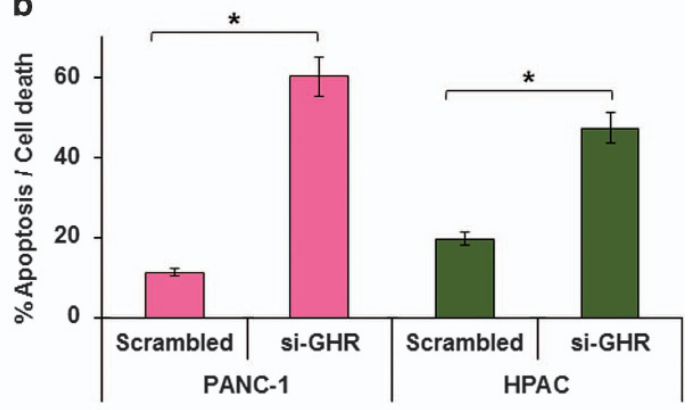

GHR inhibition induces death receptorand mitochondrial mediated apoptosis in PANC-1 \& HPAC cells

C

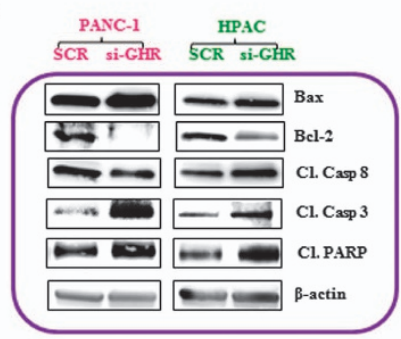

d

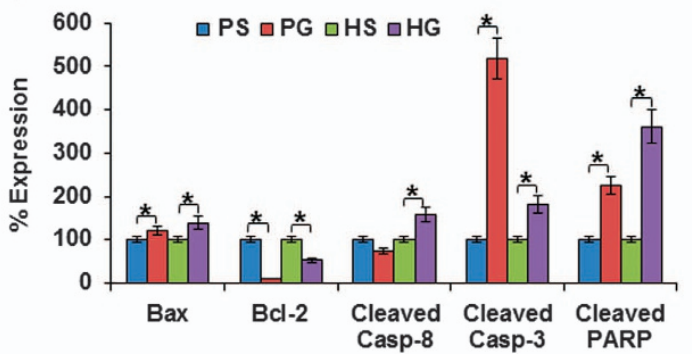

Figure 4 Apoptosis is increased in growth hormone receptor (GHR) silenced pancreatic cancer cell lines. (a) Flow cytometric analysis showed that GHR silencing induces apoptosis in PANC1 and HPAC cells. Fluorescence-activated cell sorting (FACS) histogram of cells stained with Annexin V-fluorescein isothiocyanate and propidium iodide. The percentage of early apoptotic (bottom right quadrant), apoptotic (top right quadrant), late apoptotic and necrotic cells (top left quadrant), and live healthy cells (bottom left quadrant) are represented. (b) Percentage apoptosis and cell death in PANC-1 and HPAC cells. (c) GHR inhibition induces apoptosis in PANC1 and HPAC cells. Bax, Bcl-2, caspase 8, caspase 3 and cleaved PARP and $\beta$-actin expression was assayed using western blot in GHR silenced PANC-1 and HPAC cells. (d) Densitometry analysis obtained from density ratio between apoptotic markers and $\beta$-actin shows significant potentiation of apoptosis in GHR silenced pancreatic cancer cells. Data presented as the mean \pm s.d. $(n=3)$. ${ }^{*} P<0.05$.

apoptosis and inhibits proliferation by suppressing the active status of PI3K/AKT and its intermediates.

Constitutive activation of ERK is commonly found in various primary tumors and also in PDAC. ${ }^{19}$ ERK is known to regulate many important cellular functions such as survival, proliferation, mitosis, motility, differentiation and apoptosis. ${ }^{20}$ As simultaneously targeting PI3K/AKT and ERK was shown to have an additive effect in reducing proliferation of melanoma cells, ${ }^{21}$ we sought to determine the effect of GHR on ERK signaling. GHR silenced PANC-1 and HPAC cells demonstrated a significant inhibition of ERK phosphorylation, and no change was observed in total ERK expression (Figures $6 \mathrm{a}$ and b). These results indicate that GHR functions upstream of ERK and may be a good therapeutic target for controlling the growth and progression of pancreatic cancer.

Another pathway known to be deregulated in PDAC is the JAK/STAT pathway. ${ }^{22}$ This pathway has a prominent role in initiation and progression of PDAC ${ }^{23}$ and is widely known for its role in cell proliferation, migration, invasion and apoptosis. ${ }^{24}$ STAT3 is one of the isoforms of STAT protein family that is constitutively activated in most cancers in response to growth factors and cytokines. Activated STAT3 has been shown to involve in cell proliferation and angiogenesis through the upregulation of Bcl-xl, Bcl-2, cyclin D1, c-myc, mcl-1 and VEGF. Here, we demonstrate that silencing GHR results in markedly reduced levels of STAT3 phosphorylation, as well as, reduced levels of the pro-inflammatory cytokine cyclo-oxygenease-2 (COX-2) (Figures $6 \mathrm{c}$ and d). Insulin signaling is one of the key pathways that activate STAT3 signaling and overexpression of insulin receptor- $\beta$ (IR- $\beta$ ) has been linked to cancer pathogenesis. ${ }^{25}$ Moreover, overexpression of IR- $\beta$ has been associated with suppression of PTEN. ${ }^{26}$ Thus, we assessed the expression of active and total forms of IR substrate-1 (IRS-1) and found that GHR silencing profoundly decreased the levels of phospho IRS-1 (pIRS-1) in PANC-1 and HPAC cells (Figures $6 c$ and d). Remarkably, GHR suppression also proficiently inhibited the expression of IR- $\beta$, IGF-1R and Ras (Figures $6 \mathrm{c}$ and $\mathrm{d}$ ). These results suggest that there is a significant cross-talk between GHR and the IR- $\beta$, IGF-1R and Ras signaling pathways in promoting PDAC pathogenesis.

\section{DISCUSSION}

Aberrant GHR expression is associated with increased risk of developing several types of cancer, including breast, prostate, 

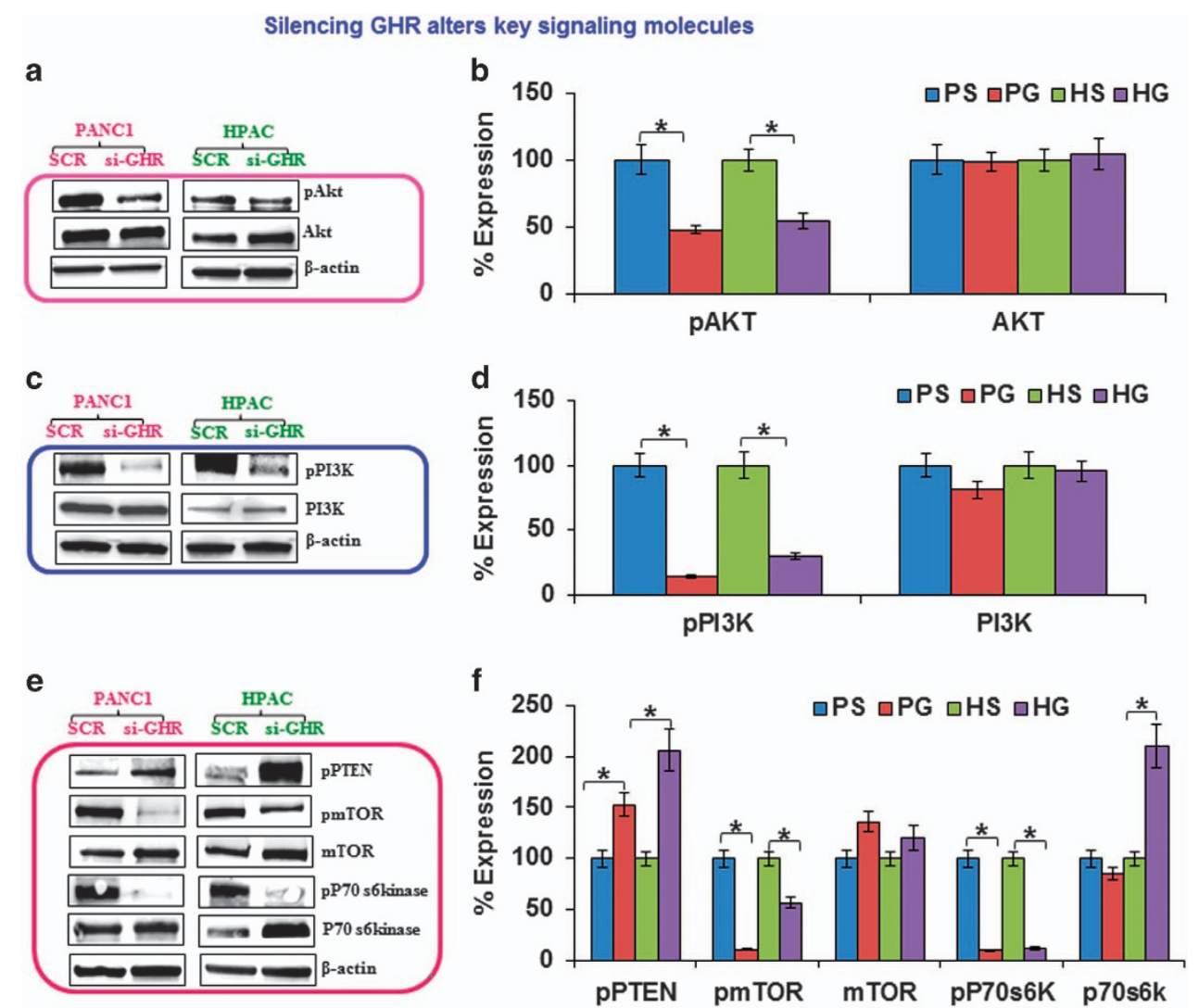

Figure 5 Suppression of growth hormone receptor (GHR) alters key signaling molecules in PANC-1 and HPAC cells. (a, c, e) The effect of GHR knockdown on AKT/phosphatidylinositol 3 kinase (PI3K) signaling was analyzed in pancreatic cancer cells. The expression of phospho-AKT, AKT, phospho-PI3K, PI3K, phospho-phosphatase and tensin homolog (PTEN), phospho-mammalian target of rapamycin (mTOR), mTOR, phospho-p70s6kinase, p70s6kinase and the internal control $\beta$-actin was measured by western blotting. (b, d, f) Densitometric analysis is also shown to the right of each representative image. Data presented as the mean \pm s.d. $(n=3)$. ${ }^{*} P<0.05$.

lung, melanoma, endometrial and colon cancer. ${ }^{27}$ The purpose of this study was to investigate the impact of silencing GHR on pancreatic cancer pathogenesis. To the best of our knowledge, this is the first study to directly assess the role of GHR in pancreatic cancer. Only two previous studies have provided indirect evidence suggesting a role for GH signaling in pancreatic cancer cell growth. ${ }^{28,29}$ However, the precise role of GHR signaling and its downstream targets is yet to be defined in pancreatic cancer pathogenesis. There is a great need to define better therapeutic targets in the treatment of PDAC because this remains one of the most understudied and fatal cancers affecting humans. ${ }^{30}$ It is already well appreciated that GH signaling via GHR has an important role in the growth of both normal and neoplastic cells. ${ }^{31}$

In many cases, it is the more aggressive and poorly differentiated cancers that tend to overexpress GHR. ${ }^{32}$ Given the fact that pancreatic cancer is one of the most aggressive and poorly differentiated cancers affecting humans, ${ }^{33}$ it is not surprising that compared with normal pancreatic cells our results reveal significant overexpression of GHR in PDAC (cell lines (PANC-1, HPAC and MIAPaCa-2) and human pancreatic adenocarcinoma tissues). Using siRNA approach, we successfully knocked down GHR in PDAC cells and clearly demonstrate for the first time that GHR has a principal role in PDAC cell proliferation, migration, invasion and thus, metastasis.

Metastatic potential is arguably the most important factor in determining how fatal a given cancer will be. ${ }^{34}$ Thus, the finding that GHR exerts significant control over PDAC metastatic potential is a strong indication that GHR has excellent therapeutic potential for the clinical treatment of PDAC. Here, not only was cell motility and invasion significantly arrested in GHR silenced PDAC cells, but the EMT was also potently inhibited. The ability to undergo EMT is a classic hallmark of cancer and is required for metastasis-both locally within the tissue of origin and distally to other parts of the body. ${ }^{35}$ Here, we show that GHR knockdown blocked the expression of the mesenchymal markers $\mathrm{N}$-cadherin, vimentin, notch-2, snail, zeb and slug, while it augmented the expression of the epithelial marker E-cadherin. The loss of E-cadherin is a hallmark of increased EMT and is adequate to confer increased metastatic potential of the cancer cells. ${ }^{16}$ This is due to structural alterations that result in decreased cell-cell junctions upon loss of E-cadherin. Therefore, silencing GHR profoundly inhibits EMT, which is the primary process involved in invasion and metastasis of PDAC cells. 
a

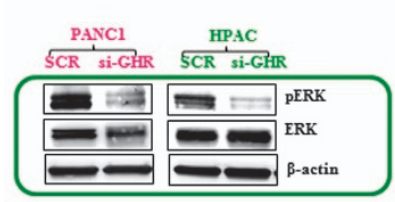

C

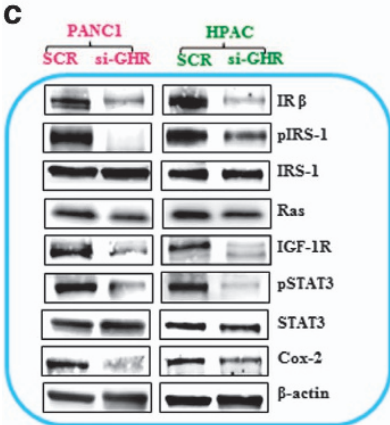

Silencing GHR alters ERK and STAT signaling in PANC-1 and HPAC cells

b
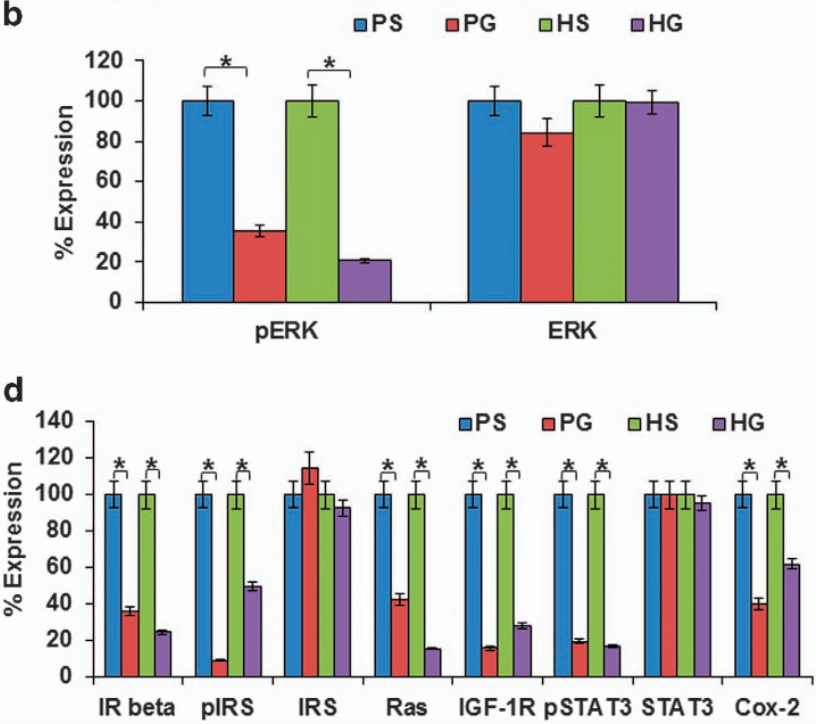

Figure 6 Silencing growth hormone receptor (GHR) alters extracellular signal-regulated kinase (ERK) and signal transducers and activators of transcription (STAT) signaling in PANC-1 and HPAC cells. Western blot analysis showing the effect of GHR silenced PANC-1 and HPAC on ERK and STAT signaling. Active and total forms of proteins were analyzed using whole-cell lysates from control and GHR silenced cells. (a, c) The expression levels of phospho-ERK, ERK, insulin receptor- $\beta$ (IR- $\beta$ ), phospho-insulin receptor substrate-1 (IRS-1), IRS, Ras, insulin-like growth factor-1 receptor (IGF-1R), phospho-STAT3, STAT3, COX-2 and $\beta$-actin. The corresponding densitometric analysis is shown to the right of each image (b and $\mathbf{d}$ ). PS-PANC-1 Scrambled, PG-PANC-1 GHR silenced, HS-HPAC Scrambled, HG-HPAC GHR silenced. Data presented as the mean \pm s.d. $(n=3)$. ${ }^{*} P<0.05$.

Our results further reveal that inhibition of GHR also causes significant reduction of cell proliferation and increased induction of apoptosis. Although apoptosis was induced in both PDAC cell lines, our results show the induction of apoptosis at a higher frequency in GHR silenced PANC-1 cells than HPAC cells. GHR silencing led to downregulation of $\mathrm{Bcl}-2$ and upregulation of Bax, caspase 3 and cleaved PARP in both cell lines, whereas caspase 8 was also upregulated in HPAC cells alone. Caspase 8 is the classical initiator molecule involved in the death receptor/extrinsic pathway of apoptosis. ${ }^{36}$ In HPAC cells, apoptosis was effected by both intrinsic and extrinsic pathways, while in PANC-1 it seems to be mainly through intrinsic pathway. These results warrant further investigation as different pancreatic cancer subtypes may respond somewhat differently to the same treatment. We believe targeting GHR will not only reduce the spread of PDAC to other sites (inhibition of metastasis), but will also effectively inhibit tumor growth through induction of apoptosis and decreased cell proliferation. Together, we expect that these effects will translate to superior disease resolution and much better life expectancy in PDAC patients.

Many studies indicate a close connection between GHR and IGF-1R signaling in the initiation and progression of multiple cancers. ${ }^{37}$ Moreover, IGF-1R is often overexpressed in pancreatic cancer where it is associated with resistance to apoptosis and exhibits increased cell proliferation. ${ }^{9}$ Similar to previous studies, we demonstrate that GHR silencing results in a reduction of IGF-1R levels in both PDAC cell lines. Further, we observed a simultaneous reduction of IR and IRS-1 levels upon GHR silencing. Inhibition of IGF-1R, IR and IRS may confer an improved anticancer effect since activation of these factors are also associated with PDAC tumorigenesis. ${ }^{38}$

GHR suppression enhances the expression of tumorsuppressor PTEN and negatively regulates the PI3K/AKT signaling, which further increases the antitumor effect of GHR silencing in PDAC cell lines. PTEN also negatively regulates mitogen extracellular signal-regulated kinase (MEK)/ ERK (MAPK) signaling via inhibiting the activity of Shc and IRS phosphorylation..$^{20}$ Suppressed levels of PTEN along with hyperactivated AKT expression are common in many cancers including PDAC. Altered expression levels of these markers are involved in deregulation of important physiological functions like proliferation, metastasis, angiogenesis, cell growth and apoptosis. $^{39}$

Active signaling between GH and its receptor, GHR, predominantly activates JAK/STAT/MAPK signaling to increase cell proliferation and survival. ${ }^{40}$ Here, GHR RNA interference effectively inhibited this downstream signaling cascade. MAPK signaling is essential for dedifferentiation of acinar cells into duct-like cells and this transition has a key role in development of pancreatic intraepithelial neoplasia. ${ }^{41}$ As mentioned above, GHR-deficient PDAC cell lines exhibited reduced pIRS-1 expression and this has been shown to suppress MEK/ERK and PI3K/AKT signaling in other systems. ${ }^{42}$ Thus, we believe that a similar mechanism is at work in PDAC cell lines where GHR silencing results in IRS-1 suppression and this ultimately leads to reduction of MEK/ERK and PI3K/AKT signaling. Indeed, we found that the active form of ERK (pERK1/2) was 
profoundly inhibited in GHR suppressed PANC-1 and HPAC cells, which is likely a major factor contributing to decreased proliferation of PDAC cells. GHR silencing strongly decreased the pAKT, pPI3K, pmTOR and also p70S6Kinase expression, which could have contributed to both increased apoptosis and decreased proliferation of PDAC cell lines. Current reports indicate that targeting mTOR itself has significant therapeutic potential for PDAC treatment. ${ }^{43}$ Taken together, our findings support the notion that silencing GHR signaling inhibits important pathways in PDAC pathogenesis, including mTOR.

Transcription factors like STAT and nuclear factor- $\kappa \mathrm{B}$ are known to upregulate COX-2 levels and increase the risk of cancer. ${ }^{44}$ Further, mutations in Kras are associated with $>90 \%$ of precancerous pancreatic intraepithelial neoplasia lesions. ${ }^{41}$ Activated Kras is known to stimulate many downstream mitogenic pathways, including JAK/STAT signaling, which results in hyperproliferation, anti-apoptotic and neoplastic transformation. ${ }^{45}$ In addition, STAT3 has been shown to be required for Kras tumorigenic transformation. Blockade of STAT3 efficiently induces apoptosis in kras mutant cancer cells. ${ }^{46}$ Carcinogenic Kras-MAPK signaling is regulated downstream of GHR, the master regulator for many signaling cascades in cancer cells. ${ }^{12}$ Kras also induces COX-2 expression in tumor cells and expression levels of COX-2 were positively correlated with extent of invasion and metastasis. ${ }^{47}$ A recent study has shown the intrinsic role of COX-2 in PDAC initiation and development through PI3K/AKT signaling. ${ }^{48}$ Even so, the precise role of COX-2 in PDAC development is not completely clear. ${ }^{48}$ To the best of our knowledge, we show for the first time that GHR is able to regulate the level of these molecules in PDAC: COX-2, Kras, STAT3, etc. Here we show GHR silencing diminishes constitutively expressed Ras, pSTAT3 and COX-2 levels in PDAC cells lines. This indicates that there is a therapeutic role for GHR in carcinogenesis of PDAC. Thus, targeting GHR alone appears to be sufficient for inhibition of most of the major signaling pathways involved in PDAC pathogenesis indicating that this is a superior therapeutic target for treatment of pancreatic cancer.

In summary, the results from our study give insight into the critical role that GHR has in pancreatic cancer and indicates that targeting GHR will be greatly beneficial for PDAC treatment. Our study demonstrates that silencing GHR tilts the balance between cell proliferation and cell death. Blocking GHR profoundly inhibited cell proliferation and anchoragedependent growth of PDAC cell lines culminating in apoptotic cell death. Blocking GHR expression also inhibited invasion and metastatic properties of aggressive PDAC cell lines through modulation of several signaling cascades downstream of GHR. This study reveals novel information related to the GHR molecular signaling cascade, which regulates most of the primary oncogenes and tumor suppressors involved in PDAC pathogenesis. We believe targeting GHR will be highly beneficial against a complex disease like PDAC, which is associated with the highest rates of mortality. In conclusion, GHR signaling represents a novel therapeutic target for superior treatment options in highly aggressive PDAC.

\section{CONFLICT OF INTEREST}

The authors declare no conflict of interest.

Author contributions: RS designed and carried out the experiments and also drafted the manuscript; RL-V assisted in drafting and editing the manuscript; SN, AA and TB helped in various experimental techniques and RL conceived the idea, designed and supervised the project and helped to draft the manuscript.

1 Tamburrino A, Piro G, Carbone C, Tortora G, Melisi D. Mechanisms of resistance to chemotherapeutic and anti-angiogenic drugs as novel targets for pancreatic cancer therapy. Front Pharmacol 2013; 4: 56.

2 Bosco G, Guizzon L, Yang Z, Camporesi E, Casarotto A, Bosio C et al. Effect of hyperbaric oxygenation and gemcitabine on apoptosis of pancreatic ductal tumor cells in vitro. Anticancer Res 2013; 33: 4827-4832.

3 Ghaneh P, Costello E, Neoptolemos JP. Biology and management of pancreatic cancer. Gut 2007; 56: 1134-1152.

4 Macgregor-Das AM, lacobuzio-Donahue CA. Molecular pathways in pancreatic carcinogenesis. J Surg Oncol 2013; 107: 8-14.

5 Zhu T, Goh EL, Graichen R, Ling L, Lobie PE. Signal transduction via the growth hormone receptor. Cell Signal 2001; 13: 599-616.

6 Banziger-Tobler NE, Halin C, Kajiya K, Detmar M. Growth hormone promotes lymphangiogenesis. Am J Pathol 2008; 173: 586-597.

7 Hayashi AA, Proud CG. The rapid activation of protein synthesis by growth hormone requires signaling through mTOR. Am J Physiol Endocrinol Metab 2007; 292: E1647-E1655.

8 Kopchick JJ, Andry JM. Growth hormone (GH), GH receptor, and signal transduction. Mol Genet Metab 2000; 71: 293-314.

9 Nair PN, De Armond DT, Adamo ML, Strodel WE, Freeman JW. Aberrant expression and activation of insulin-like growth factor-1 receptor (IGF-1R) are mediated by an induction of IGF-1R promoter activity and stabilization of IGF-1R mRNA and contributes to growth factor independence and increased survival of the pancreatic cancer cell line MIA PaCa-2. Oncogene 2001; 20: 8203-8214.

10 Bustin SA, Jenkins PJ. The growth hormone-insulin-like growth factor-I axis and colorectal cancer. Trends Mol Med 2001; 7: 447-454.

11 Sustarsic EG, Junnila RK, Kopchick JJ. Human metastatic melanoma cell lines express high levels of growth hormone receptor and respond to $\mathrm{GH}$ treatment. Biochem Biophys Res Commun 2013; 441: 144-150.

12 Clayton PE, Banerjee I, Murray PG, Renehan AG. Growth hormone, the insulin-like growth factor axis, insulin and cancer risk. Nat Rev Endocrinol 2011; 7: 11-24.

13 Qin B, Cheng K. Silencing of the IKKepsilon gene by siRNA inhibits invasiveness and growth of breast cancer cells. Breast Cancer Res 2010; 12: R74.

14 Thiery JP. Epithelial-mesenchymal transitions in tumour progression. Nat Rev Cancer 2002; 2: 442-454.

15 Chaffer CL, Weinberg RA. A perspective on cancer cell metastasis. Science 2011; 331: 1559-1564.

16 Onder TT, Gupta PB, Mani SA, Yang J, Lander ES, Weinberg RA. Loss of E-cadherin promotes metastasis via multiple downstream transcriptional pathways. Cancer Res 2008; 68: 3645-3654.

17 Page C, Lin HJ, Jin Y, Castle VP, Nunez G, Huang M et al. Overexpression of Akt/AKT can modulate chemotherapy-induced apoptosis. Anticancer Res 2000; 20: 407-416.

18 Garcia-Carracedo D, Turk AT, Fine SA, Akhavan N, Tweel BC, Parsons R et al. Loss of PTEN expression is associated with poor prognosis in patients with intraductal papillary mucinous neoplasms of the pancreas. Clin Cancer Res 2013; 19: 6830-6841.

19 Davies H, Hunter C, Smith R, Stephens P, Greenman C, Bignell G et al. Somatic mutations of the protein kinase gene family in human lung cancer. Cancer Res 2005; 65: 7591-7595.

20 Roy SK, Srivastava RK, Shankar S. Inhibition of PI3K/AKT and MAPK/ERK pathways causes activation of FOXO transcription factor, leading to cel 
cycle arrest and apoptosis in pancreatic cancer. J Mol Signal 2010; 5(10): 2187-5-10.

21 Babchia N, Calipel A, Mouriaux F, Faussat AM, Mascarelli F. The PI3K/Akt and mTOR/P70S6K signaling pathways in human uveal melanoma cells: interaction with B-Raf/ERK. Invest Ophthalmol Vis Sci 2010; 51: 421-429.

22 Jones S, Zhang X, Parsons DW, Lin JC, Leary RJ, Angenendt P et al. Core signaling pathways in human pancreatic cancers revealed by global genomic analyses. Science 2008; 321: 1801-1806.

23 Palagani V, Bozko P, El Khatib M, Belahmer H, Giese N, Sipos B et al. Combined inhibition of Notch and JAK/STAT is superior to monotherapies and impairs pancreatic cancer progression. Carcinogenesis 2014; 35 : 859-866.

24 Mughal TI, Girnius S, Rosen ST, Kumar S, Wiestner A, Abdel-Wahab 0 et al. Emerging therapeutic paradigms to target the dysregulated JAK/STAT pathways in hematological malignancies. Leuk Lymphoma 2014; 55: 1968-1979.

25 Belfiore A, Malaguarnera R. Insulin receptor and cancer. Endocr Relat Cancer 2011; 18: R125-R147.

26 Liu J, Visser-Grieve S, Boudreau J, Yeung B, Lo S, Chamberlain G et al. Insulin activates the insulin receptor to downregulate the PTEN tumour suppressor. Oncogene 2013. 1-8.

27 Perry JK, Liu DX, Wu ZS, Zhu T, Lobie PE. Growth hormone and cancer: an update on progress. Curr Opin Endocrinol Diabetes Obes 2013; 20: 307-313.

28 Harrison LE, Blumberg D, Berman R, Ng B, Hochwald S, Brennan MF et al. Effect of human growth hormone on human pancreatic carcinoma growth, protein, and cell cycle kinetics. J Surg Res 1996; 61: 317-322.

29 Szepeshazi K, Schally AV, Groot K, Armatis P, Hebert F, Halmos G. Antagonists of growth hormone-releasing hormone $(\mathrm{GH}-\mathrm{RH})$ inhibit in vivo proliferation of experimental pancreatic cancers and decrease IGF-II levels in tumours. Eur J Cancer 2000; 36: 128-136.

30 Clark KL, Loscalzo M, Trask PC, Zabora J, Philip EJ. Psychological distress in patients with pancreatic cancer-an understudied group. Psychooncology 2010; 19: 1313-1320.

31 Friend KE. Cancer and the potential place for growth hormone receptor antagonist therapy. Growth Horm IGF Res 2001; 11(Suppl A): S121-S123.

32 Brown RJ, Adams JJ, Pelekanos RA, Wan Y, McKinstry WJ, Palethorpe K et al. Model for growth hormone receptor activation based on subunit rotation within a receptor dimer. Nat Struct Mol Biol 2005; 12: 814-821.

33 Wang Z, Li Y, Ahmad A, Banerjee S, Azmi AS, Kong D et al. Pancreatic cancer: understanding and overcoming chemoresistance. Nat Rev Gastroenterol Hepatol 2011; 8: 27-33.

34 Verburg FA, Mader U, Tanase K, Thies ED, Diessl S, Buck AK et al. Life expectancy is reduced in differentiated thyroid cancer patients $>/=45$ years old with extensive local tumor invasion, lateral lymph node, or distant metastases at diagnosis and normal in all other DTC patients. J Clin Endocrinol Metab 2013; 98: 172-180.

35 Schneider D, Baronsky T, Pietuch A, Rother J, Oelkers M, Fichtner D et al. Tension monitoring during epithelial-to-mesenchymal transition links the switch of phenotype to expression of moesin and cadherins in NMuMG cells. PLoS One 2013; 8: e80068.
36 Luo X, Budihardjo I, Zou H, Slaughter C, Wang X. Bid, a Bcl2 interacting protein, mediates cytochrome $c$ release from mitochondria in response to activation of cell surface death receptors. Cell 1998; 94: 481-490.

37 Gallagher EJ, LeRoith D. Is growth hormone resistance/IGF-1 reduction good for you? Cell Metab 2011; 13: 355-356.

38 Chen HX, Sharon E. IGF-1R as an anti-cancer target-trials and tribulations. Chin J Cancer 2013; 32: 242-252.

39 Yao Z, Okabayashi Y, Yutsudo Y, Kitamura T, Ogawa W, Kasuga M. Role of Akt in growth and survival of PANC-1 pancreatic cancer cells. Pancreas 2002; 24: 42-46.

40 Sedek M, van der Velden LM, Strous GJ. Multimeric growth hormone receptor complexes serve as signaling platforms. J Biol Chem 2014; 289: 65-73.

41 Collins MA, Yan W, Sebolt-Leopold JS, Pasca di Magliano M. Mapk signaling is required for dedifferentiation of acinar cells and development of pancreatic intraepithelial neoplasia in mice. Gastroenterology 2013; 146: 822-834.

42 Weng LP, Smith WM, Brown JL, Eng C. PTEN inhibits insulin-stimulated MEK/MAPK activation and cell growth by blocking IRS-1 phosphorylation and IRS-1/Grb-2/Sos complex formation in a breast cancer model. Hum Mol Genet 2001; 10: 605-616.

43 Silvestris N, Gnoni A, Brunetti AE, Vincenti L, Santini D, Tonini G et al. Target therapies in pancreatic carcinoma. Curr Med Chem 2014; 21 : 948-965.

44 Khan S, Shukla S, Sinha S, Meeran SM. Role of adipokines and cytokines in obesity-associated breast cancer: therapeutic targets. Cytokine Growth Factor Rev 2013; 24: 503-513.

45 Adjei AA. Blocking oncogenic Ras signaling for cancer therapy. J Natl Cancer Inst 2001; 93: 1062-1074.

46 Park E, Park J, Han SW, Im SA, Kim TY, Oh DY et al. NVP-BKM120, a novel PI3K inhibitor, shows synergism with a STAT3 inhibitor in human gastric cancer cells harboring KRAS mutations. Int J Oncol 2012; 40: 1259-1266

47 Li M, Liu W, Zhu YF, Chen YL, Zhang BZ, Wang R. Correlation of COX-2 and K-ras expression to clinical outcome in gastric cancer. Acta Oncol 2006; 45: 1115-1119.

48 Hill R, Li Y, Tran LM, Dry S, Calvopina JH, Garcia A et al. Cell intrinsic role of COX-2 in pancreatic cancer development. Mol Cancer Ther 2012; 11 : 2127-2137.

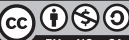

This work is licensed under a Creative Commons Attribution-NonCommercial-ShareAlike 3.0 Unported License. The images or other third party material in this article are included in the article's Creative Commons license, unless indicated otherwise in the credit line; if the material is not included under the Creative Commons license, users will need to obtain permission from the license holder to reproduce the material. To view a copy of this license, visit http:// creativecommons.org/licenses/by-nc-sa/3.0/ 